\title{
TOPOLOGIA VIRTUAL E TOPOLOGIA FÍSICA DE REDES ÓPTICAS: UMA PROPOSTA DE PROJETO INTEGRADO*
}

\author{
Karcius D. R. Assis e Helio Waldman
}

\begin{abstract}
Resumo - As estratégias tradicionais para o projeto de redes ópticas roteadoras de comprimento de onda fazem uma divisão do projeto através de dois problemas: Projeto da Topologia Virtual (VTD) em que conexões virtuais são derivadas da demanda de tráfego e Roteamento e Alocação de Comprimento de Onda (RWA), em que os caminhos ópticos são acomodados na topologia física para suportar a requisição de conexões. Nós propomos um algoritmo iterativo através de programação linear, para resolver os dois problemas de forma integrada, através de diversas funções objetivo: congestionamento, carga e pool de comprimentos de onda. A solução do VTD gera requisições para um conjunto de caminhos que devem ser acomodados na topologia física. Os caminhos são então alocados de maneira a minimizar critérios de otimização através da estratégia iterativa.
\end{abstract}

Palavras-chave: Redes ópticas, roteamento e alocação de comprimento de onda, projeto da topologia virtual.

\begin{abstract}
Traditional approaches to wavelength routing network design divide it into two separate problems: virtual topology design (VTD), in which best connections among nodes are derived from traffic demand; and routing-andwavelength assignment (RWA), in which physical paths are accommodated in the physical topology to support the requested connections. We propose an iterative linear programming approach to solve both problems jointly under multiple objectives such as congestion avoidance, fiber load and wavelength pool minimization. The solution of the VTD problem generates a request for a set of paths to be supplied by the physical topology. Physical paths are then allocated in order to minimize some objective function that is akin to a linear programming formulation.
\end{abstract}

Keywords: Optical networks, routing and wavelength assignment, virtual topology design.

\section{INTRODUÇÃO}

A transmissão de informação por meio de fibras ópticas cresceu e amadureceu enormemente nos últimos 30 anos; e segundo critérios técnicos e econômicos, é a opção mais atraente para as redes de comunicações com serviços de faixa larga. Avanços recentes nesta área vêm revolucionando a

Karcius faz parte do Núcleo de Pesquisa Interdepartamental em Redes (NUPERC) da UNIFACS, Salvador, BA, Brasil. H. Waldman faz parte do Optical Networking Lab. do Departamento de Comunicações da FEEC/Unicamp, Campinas, SP, Brasil.

E-mails: karcius@unifacs.br, waldman@decom.fee.unicamp.br. Editor de Área responsável: Nelson Fonseca. Artigo submetido em 06/Mai/2004, revisado em 30/Jun/2004, aceito em 08/Jul/2004. infra-estrutura de telecomunicações em todo o mundo. Capacidades de transmissão cada vez mais elevadas (até 40 Gbps em cada canal) têm possibilitado a implantação de redes de comunicações que viabilizam a integração de diferentes tipos de tráfego, a sofisticação dos serviços e a interconexão de redes remotas [1].

Paradoxalmente, desde a invenção do computador até os anos 80 , as velocidades de processamento vieram aumentando bem mais rapidamente do que as velocidades de transmissão. Com isso a transmissão era o gargalo das redes de comunicações. No entanto, a partir dos anos $80 \mathrm{com}$ o amadurecimento da tecnologia da transmissão óptica digital, as velocidades de transmissão tiveram um aumento de várias ordens de magnitude, deslocando o gargalo para os nós eletrônicos de processamento [2]. Este eletronic botlenneck é demonstrado pela "Lei Empírica de Moore" [3].

A tecnologia mais viável, até o momento, para superar esse descompasso, é a multiplexagem por divisão de comprimento de onda (WDM), que consiste em modular eletronicamente vários comprimentos de onda por sinais distintos e propagálos todos ao mesmo tempo na fibra óptica constituindo a arquitetura chamada rede óptica por divisão em comprimento de onda [4]-[6].

Na primeira metade da década de 90 , a busca destas arquiteturas se concentrou nos protocolos de acesso ao meio de transmissão (MAC), já que os recursos tecnológicos disponíveis só permitiam a construção de topologias físicas do tipo broadcast, gerando as chamadas redes broadcastand-select. Já na segunda metade da década, o advento de dispositivos com capacidade de roteamento de comprimentos de onda deslocou o foco das pesquisas para as redes de caminhos ópticos [4], [11]-[15].

A rede de caminhos ópticos está sendo viabilizada com o advento dos ADM's (add-drop multiplexers) e crossconnects ópticos [4]. Com esse fim, vários produtos estão sendo lançados por grandes consórcios industriais, bem como as técnicas de gerenciamento e proteção adequadas para o tipo de tráfego a ser atendido.

O tráfego a ser atendido poderá vir da rede síncrona de transporte (padrões SONET ou SDH), que já conta com seus próprios mecanismos de proteção; ou diretamente da rede ATM, que precisa garantir qualidade-de-serviço para algumas de suas classes; ou de IP (Protocolo Internet), que não implica em garantia de qualidade-de-serviço. Por isso, diferentes caminhos ópticos podem ter diferentes requisitos de proteção, com possíveis implicações sobre a topologia física e a de caminhos físicos.

$O$ advento da camada fotônica de transporte está marcando a primeira década do século XXI assim como o padrão ATM

\footnotetext{
* Esta é uma versão estendida do artigo apresentado no IEEE/ITS'02.
} 
marcou os anos 90 . Ela é necessária para evitar os altos custos de fazer subir todo o tráfego passante ao domínio elétrico para roteá-lo em nós SDH, que são muito caros e, portanto, devem ser minimizados. Esta minimização, por sua vez, agrava o problema de engenharia de tráfego, uma vez que os comprimentos de onda passantes não poderão entregar tráfego eventualmente destinado a usuários locais (também conhecida como topologia virtual [4]), que não corresponderá mais à topologia física da rede. Otimizar o encaminhamento do tráfego sob essas condições é o problema do traffic grooming [5].

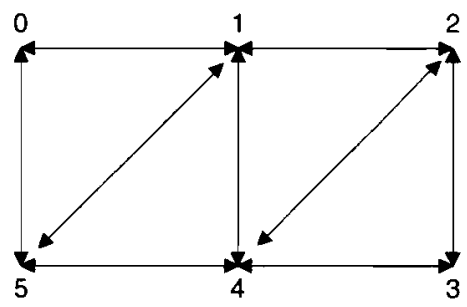

Figura 1. Exemplo ilustrativo: topologia física.

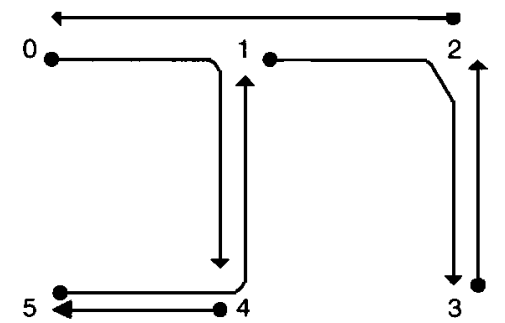

Figura 2. Exemplo Ilustrativo: topologia virtual.

O desenvolvimento da camada fotônica de transporte depende da disponibilidade de novos elementos de rede tais como filtros ópticos, acopladores, amplificadores ópticos, roteadores, conversores de comprimento de onda; e do desempenho destes elementos. A evolução destas tecnologias deverá condicionar as soluções arquitetônicas que serão adotadas pelas redes futuras. A fim de compreender este condicionamento e, por conseguinte, avaliar o impacto da tecnologia disponível sobre o desempenho sistêmico, é necessário realizar estudos sobre planejamento e projeto de redes ópticas, o qual nós realizamos neste artigo.

O enfoque principal deste artigo está voltado para o planejamento e projeto de redes ópticas que utilizam a tecnologia WDM. Especificamente os problema de projeto da topologia virtual e topologia física de forma integrada, diferentemente da literatura prévia que considera estes problemas de forma separada [6], [8]. Neste artigo damos ênfase a modelos de Programação Linear Inteira na fase de planejamento destas redes e propomos uma heurística iterativa para otimizar este planejamento.

\section{PROJETOS DA TOPOLOGIA VIRTUAL E DA TOPOLOGIA FÍSICA}

Uma topologia física $G_{p}$ é um grafo em que os arcos re- presentam um par de fibras (um em cada direção) e os nós representam nós roteadores de comprimento de onda (p.ex, roteadores IP, nós SDH etc). A Figura 1 mostra uma topologia física de uma rede de longa cobertura de seis nós. Os nós roteadores de comprimento de onda são numerados de 0 a 5 . O conjunto de todos os caminhos ópticos unidirecionais ao longo dos roteadores forma a topologia virtual $G_{v}$ ou topologia de caminhos ópticos. Por exemplo, a Figura 2 mostra uma possível interconexão virtual. Entretanto, note que o roteamento pela camada física não é visível explicitamente na topologia virtual (a impressão de que os links virtuais estão atravessando os links físicos pela topologia física da Figura 1 é feita para facilidade de compreensão). Há um link virtual na topologia virtual entre os nós 2 e 0 , o que significa que os dados ou pacotes atravessam o link virtual de 2 para 0 apenas no domínio óptico, ou seja, não há conversão ópticaeletrônica-óptica nos nós roteadores intermediários (consideramos os nós equipados com optical crossconnect (OXC)). Arcos na topologia virtual são chamados links virtuais (caminhos ópticos) e são definidos somente por seus nós fonte e destino [6].

A topologia virtual pode ser especificada por variáveis inteiras denominadas $b_{i j}$, com $i=1,2 \ldots N ; j=1,2 \ldots N$, onde $N$ é o número de nós da rede. Nós temos $b_{i j}=1$, se há um link virtual de um nó $i$ para um nó $j$; caso contrário $b_{i j}=0$. Por exemplo, na Figura 2 dados são enviados do nó 2 para o nó 0 em um único caminho óptico atravessando o nó roteador 1 ; então $b_{20}=1$. Simultaneamente, nós podemos enviar pacotes do nó 1 para o nó 3 passando pelo nó intermediário 2 . Nós observamos que na topologia física existe uma fibra conectando os nós 2 e 4 . No entanto, para enviar pacotes do nó 2 para o nó 4 nós devemos usar 2 links virtuais (ou caminhos ópticos) 2-0 e 0-4. Então, a conexão lógica tem dois hops virtuais. De maneira semelhante, nós dizemos que o número de hops físicos do link virtual $b_{20}$ é 2 , pois ele atravessa dois links físicos $(2,1)$ e $(1,0)$ na topologia física.

Para uma rede com $N$ nós, o ideal seria configurar caminhos ópticos para todos os $N(N-1)$ pares. Entretanto, isso não é usualmente possível por duas razões: Primeiro, o número de comprimentos de onda disponíveis impõe um limite na quantidade de caminhos ópticos que podem ser configurados (isto é também uma função da distribuição de tráfego). Segundo, cada nó pode ser fonte e destino de um número limitado de caminhos ópticos. Isto é determinado pela quantidade de hardware óptico que pode ser provido (transmissores e receptores) e pela quantidade total de informações que um nó pode processar [7].

Então, sendo $\mathrm{T}=\left(\lambda^{s d}\right)$ uma matriz de tráfego, i.e., $\lambda^{s d}$ é a taxa de pacotes (ou $\mathrm{Gb} / \mathrm{s}$ ) de um nó $s$ que são enviados para o nó $d$. Nós tentamos criar uma topologia virtual $G_{v}$ e rotear o tráfego nesta $G_{v}$ minimizando $\lambda_{\max }=\max \left\{\lambda_{i j}\right\}$, onde $\lambda_{i j}$ é a carga oferecida ao link $(i-j)$ da topologia virtual. A variável $\lambda_{\max }$ é a máxima carga que atravessa um link virtual e é definida como "congestionamento". Sendo $G_{p}$ a topologia física da rede, $\Delta$ o grau da topologia virtual (número de portos) e $W$ o número de comprimentos de ondas disponíveis. Uma descrição informal do problema de projeto da topologia virtual, conhecido como VTD, e topologia física, conhecido como PTD, é dada a seguir (uma 
formulação precisa usando Programação Linear Inteira Mista (MILP) será dada na próxima seção):

$$
\operatorname{Min} \lambda_{\max }
$$

Sujeito a:

- Cada link em $G_{v}$, corresponde a um caminho óptico e dois caminhos ópticos que compartilham um arco na topologia física devem ter comprimentos de ondas diferentes;

- O número total de comprimentos usados é no máximo $W$;

- Todos os nós em $G_{v}$ têm $\Delta$ arcos de entrada e $\Delta$ arcos de saída;

- O fluxo de tráfego de cada par fonte-destino é conservado nos nós intermediários.

\section{FORMULAÇÃO MATEMÁTICA PARA O VTD E PTD}

Nós formulamos os subproblemas de projeto da topologia virtual (VTD) e projeto da topologia física (PTD) como um problema de otimização através de Programação Linear Inteira Mista [6], [8], [10].

Notação:

- $s$ e $d$ denotam, respectivamente, a fonte e o destino de pacotes;

- $i$ e $j$ são os nós de origem e término, respectivamente, de um caminho óptico;

- $m$ e $n$ denotam links físicos de $m$ para $n$, nos quais podem passar um ou mais caminhos ópticos.

Dado:

- Número de nós na rede: $N$;

- Número de comprimentos de onda disponíveis: $W$;

- Topologia física $\left(P_{m n}\right)$ : Denota o número de fibras interconectando os nós $m$ e $n . P_{m n}=0$ para um nó $m$ que não é fisicamente adjacente a um nó $n . P_{m n}=P_{n m}$ indica que há igual número de fibras entre os nós $m$ e $n$, adjacentes, em direções opostas. Note que pode haver mais que um link de fibra conectando nós adjacentes na rede. Neste artigo, caso não se especifique outra definição para os links físicos, considere a rede $\operatorname{com} P_{m n}=P_{n m}=1$;

- Número de transmissores: Em um nó $i, T_{i}\left(T_{i} \quad \geq 1\right)$ é o número de transmissores deste nó e o número de receptores neste nó ié $R_{i}\left(R_{i} \geq 1\right)$. Em geral assumese $T_{i}=R_{i}$. (Estes são os graus virtuais (ou lógicos) da rede), também se usa a notação $\Delta$ (número de portos);

- Matriz de tráfego $\mathrm{T}=\left(\lambda^{s d}\right)$ : Denota a taxa média de fluxo de tráfego (em pacotes ou $\mathrm{Gb} / \mathrm{segundo}$ ) de um nó $s$ para um nó $d$, para $s, d=1,2, \ldots, N$ e com $\lambda^{s s}=0$;
- Capacidade de cada canal: $C$ (normalmente expressa em Gbits/segundo, mas convertida para unidades de pacotes por segundo, supondo conhecido o comprimento do pacote).

\section{Variáveis:}

- Topologia Virtual: A variável inteira $b_{i j}$ denota o número de caminhos ópticos de um nó $i$ para um nó $j$ na topologia virtual. Note que nesta formulação os caminhos ópticos não são necessariamente bidirecionais, isto é, $b_{i j}=0$ não implica em $b_{j i}=0$. No entanto, podemos ter múltiplos caminhos ópticos de uma fonte para um destino, se $b_{i j i} 1$; servindo para o caso em que o tráfego entre os nós $i$ e $j$ é maior que a capacidade $C$ de um único caminho óptico;

- Roteamento do tráfego: As variáveis $\lambda_{i j}^{s d}$ denotam a quantidade de tráfego fluindo de uma fonte $\mathrm{s}$ para um destino $d$, e passando por links virtuais $b_{i j}$ intermediários. Note que o tráfego de uma fonte $s$ para um destino $d$ pode ser "bifurcado", em diferentes componentes de fluxo, passando por diferentes conjuntos de caminhos ópticos;

- Carga em um link físico: $L$, sendo $L_{i}=W$. A carga denota o número máximo de caminhos ópticos que devem atravessar um link físico da rede;

- Roteamento na topologia física: A variável $p_{m n}^{i j}$ denota o caminho óptico entre os nós $i$ e jque está sendo roteado pelo link de fibra $m-n$;

- Alocação de comprimento de onda: A variável $p_{m n \varsigma}^{i j}$ especifica o comprimento de onda $\varsigma$ que é alocado ao caminhos óptico entre os nós $m$ e $n$ da topologia física. Como não há conversão de comprimento de onda, esse mesmo valor será alocado para todos os links físicos por qual o correspondente caminho óptico $i-j$ passa.

Logo, a formulação matemática através de Programação Linear Inteira Mista (MILP) para os problemas VTD e PTD são dadas por:

VTD-Virtual Topology Design:

$$
\text { Minimize : } \lambda_{\max } \text {. }
$$

A Função objetivo é minimizar o congestionamento na rede (em termos de pacotes/segundo que atravessam um link virtual)

Sujeito a:

- Conservação de fluxo em cada nó:

$$
\sum_{j} \lambda_{i j}^{s d}-\sum_{j} \lambda_{j i}^{s d}=\left\{\begin{array}{rll}
\lambda^{s d} & \text { se } & s=i \\
-\lambda^{s d} & \text { se } & d=i \\
0 & \text { c.c } &
\end{array}\right.
$$

- Fluxo total em um link virtual:

$$
\begin{aligned}
& \lambda_{i j}=\sum_{s d} \lambda_{i j}^{s d} \\
& \lambda_{i j} \leq \lambda_{\max } \\
& \lambda_{i j}^{s d} \leq b_{i j} \lambda^{s d}
\end{aligned}
$$


Equações (2)-(4) são equações de fluxo multicommodity que governam o tráfego na topologia virtual. Note que o tráfego de uma fonte para um destino pode ser "bifurcado". As equações (5) e (6) são definições da variável congestionamento. Equação (7) garante que o tráfego só pode fluir por um caminho óptico existente.

- Matriz de conexão $b_{i j}$ da topologia virtual:

$$
\begin{aligned}
& \sum_{j} b_{i j} \leq \Delta=T_{i}, \quad \forall_{i} \\
& \sum_{i} b_{i j} \leq \Delta=R_{j}, \quad \forall_{j}
\end{aligned}
$$

As restrições acima, (8) e (9), asseguram que o número de caminhos ópticos emergindo de um nó é restrito ao número transmissores nesse nó, enquanto que o número de caminhos ópticos que terminam em um nó é restrito ao número de receptores nesse nó.

- Restrições de não-negatividade e número inteiros:

$$
\begin{gathered}
\lambda_{i j}^{s d}, \lambda_{i j}, \lambda_{\max } \geq 0 \\
b_{i j} \in\{0,1\}
\end{gathered}
$$

\section{PTD-Phisical Topology Design:}

- Roteamento na topologia física $p_{m n}^{i j}$ :

$$
\begin{aligned}
& \sum_{m} p_{m k}^{i j}=\sum_{n} p_{k n}^{i j}, \quad \text { se } k \neq i, j \\
& \sum_{n} p_{i n}^{i j}=b_{i j} \\
& \sum_{m} p_{m j}^{i j}=b_{i j} \\
& \sum_{i j} p_{m n}^{i j} \leq L . P_{m n} .
\end{aligned}
$$

As equações (10)-(12) garantem o roteamento dos caminhos ópticos $\left(b_{i j}\right)$ na topologia física através de equações de fluxo multicommodity. Note que o commodity agora é um caminho óptico e não "dados" como em (2)-(4). A equação (13) limita o número máximo de caminhos ópticos que atravessam um link físico através da variável carga $L$.

- Alocação de comprimento de onda e restrições de continuidade de comprimento de onda (sem conversão em nenhum nó):

$$
\begin{aligned}
& \sum_{m} p_{m \varsigma \varsigma}^{i j}=\sum_{n} p_{\ln \varsigma}^{i j} \quad \text { se } l \neq i, j \\
& \sum_{n} p_{i n \varsigma}^{i j}=b_{i j \varsigma} \\
& \sum_{m} p_{m j \varsigma}^{i j}=b_{i j \varsigma} \\
& \sum_{\varsigma} b_{i j \varsigma}=b_{i j} \\
& \sum_{i j} p_{m n \varsigma}^{i j} \leq P_{m n} \\
& \sum_{\varsigma} p_{m n \varsigma}^{i j}=p_{m n}^{i j}
\end{aligned}
$$

As equações (14)-(19) permitem a alocação adequada de comprimentos de ondas para os caminhos ópticos roteados na topologia física. Note que as restrições não permitem conversão de comprimento de onda nos nós intermediários da rede.

- Condição de não-negatividade e número inteiros:

$$
\text { int } p_{m n}^{i j}, p_{m n \varsigma}^{i j} \text { e } p_{m n}^{i j} \geq 0, p_{m n \varsigma}^{i j} \geq 0
$$

\section{ESTRATÉGIA HEURÍSTICA}

Os problemas VTD e PTD descritos anteriormente são complexos e cada um deles é conhecido como NP-Completo [5], ou seja, o tempo de execução do problema cresce de maneira exponencial quando o número de variáveis aumenta. Logo, se cada um dos problemas é NP, a solução dos dois conjuntamente é ainda mais complexa. Então, a divisão em VTD e PTD é aceitável porque diminui esta complexidade. Entretanto, para uma maior eficiência do uso dos recursos da rede sob uma perspectiva de integração dos planos de controle da camada óptica (PTD) e da camada cliente (VTD), o interessante é encontrar uma maneira para resolver estes problemas de forma integrada e num tempo computacional aceitável. Para isto, buscamos uma estratégia para tornar o problema com muitas restrições tratável. Uma proposta é mostrada no algoritmo heurístico da Figura 3, chamado MinW, onde o problema foi dividido em subproblemas, onde cada um gera uma entrada para o subproblema posterior, a qual pode ser viável ou não.

Note que resolver o projeto da topologia virtual é equivalente a achar os valores das variáveis $b_{i j}$, resolver o projeto da topologia física é equivalente a achar os valores das variáveis $p_{m n}^{i j}$, e resolver o problema de coloração de grafos (alocação de comprimentos de onda) significa achar as variáveis $p_{m n \varsigma}^{i j}$. Se cada qual, separadamente, resultar em soluções viáveis, o problema é resolvido completamente. Caso contrário, se um dos subproblemas é inviável, deve-se projetar uma nova topologia virtual e executar o algoritmo novamente.

É interessante observar ainda que cada um dos problemas acima deve ser formulado com vistas à otimização de uma função objetivo diferente. O problema da topologia virtual, conforme expresso em (1) será formulado para minimizar o congestionamento na rede, o que equivale à maximização de eficiência de recursos na rede, bem como a minimização do processamento eletrônico intermediário. Já o projeto da topologia física poderia ter como objetivo minimizar a carga $L$, e o problema de coloração de grafo, se houver restrição de continuidade de comprimento de onda, minimizar $W$ (\# de comprimentos de onda). A viabilidade da solução seria caracterizada pela conformidade dos valores mínimos de $L$ e $W$ à carga máxima das fibras e à cardinalidade do "pool" de comprimentos de onda, respectivamente.

Cada vez que for constatada a inviabilidade da solução da topologia física ou da coloração, o retorno ao problema da topologia virtual pede a próxima melhor solução, após as que se mostrarem inviáveis. $\mathrm{O}$ algoritmo usado para a solução do VTD, portanto, deve ser capaz de listar soluções para os $b_{i j}$ por ordem de aproximação ao ótimo da função objetivo. 


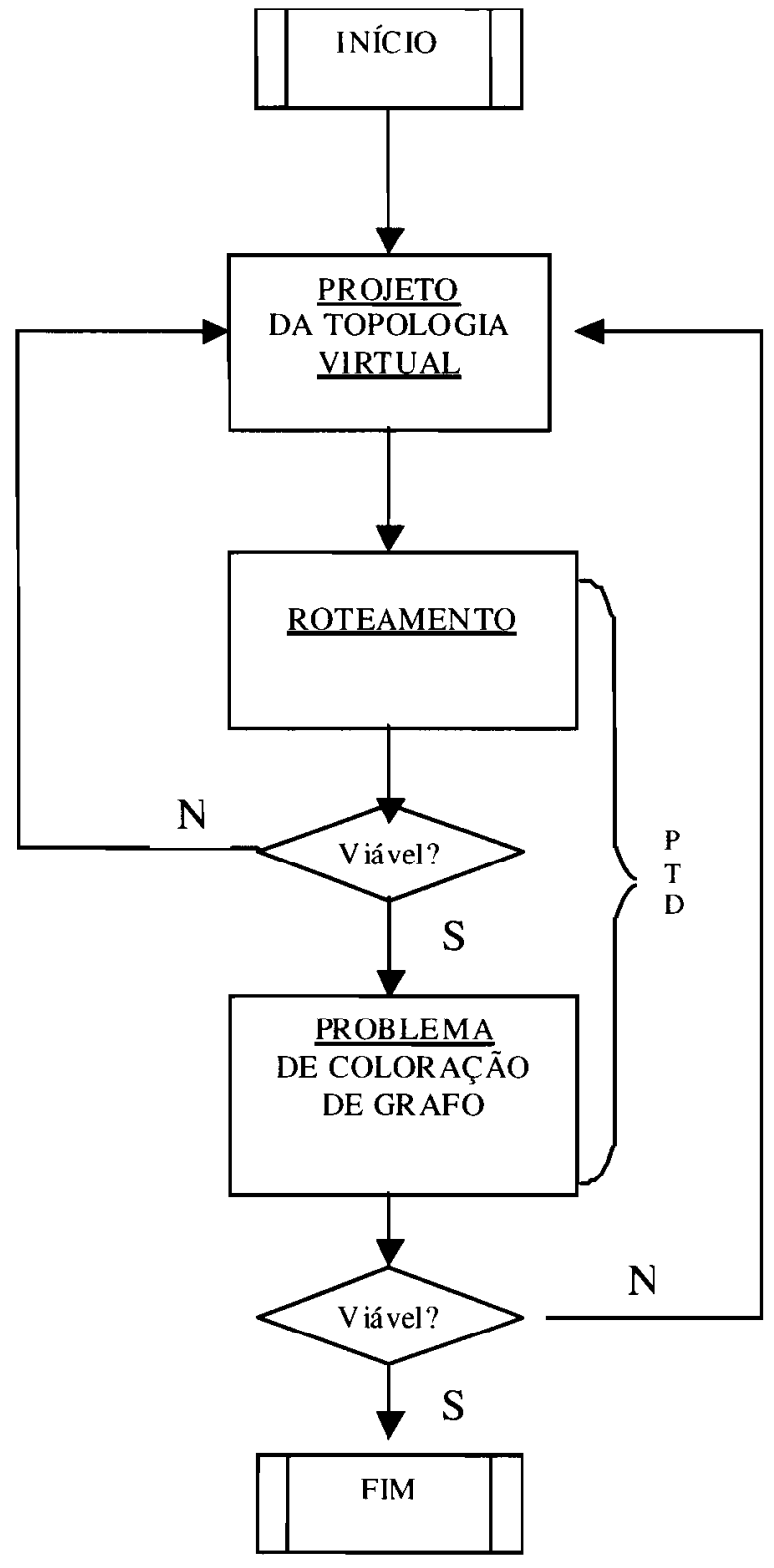

Figura 3. Algoritmo MinW.

\section{SIMULAÇÕES}

Para simulações, usamos a estratégia descrita na seção anterior da seguinte maneira:

1. VTD: De maneira a aliviar a complexidade computacional associada ao MILP, utilizamos a tradicional heurística HVTD (Heuristic for the Virtual Topology Design, [6]) neste passo; para assim encontrar os $b_{i j}$ de uma dada matriz de tráfego. A heurística tenta estabelecer caminhos ópticos entre os pares fonte-destino com maiores valores de $\mathrm{T}=\lambda^{s d}$, e é sujeita a restrição do grau virtual. Nós decidimos resolver o VTD pela heurística HVTD, abandonando as equações (1)-(9), porque a HVTD é uma heurística clássica [6]. Entretanto, o tráfego pode ser facilmente roteado pelos $b_{i j}$ encontados neste passo, por equações de fluxo multicommodity [8].
2. PTD: Resolver (10)-(13) com a função objetivo:

$$
\text { Min } L \text {. }
$$

Nosso objetivo aqui é minimizar a máxima carga necessária em qualquer link de fibra da rede, de maneira a estabelecer o conjunto de caminhos ópticos na topologia física da rede. Este passo dá uma solução temporária para ser validada no próximo passo.

3. Coloração: Dado $L$, re-otimize (10)-(19) com a função objetivo

$$
\operatorname{Min} \sum_{m n i j} p_{m n}^{i j} .
$$

Isto é necessário porque a minimização da carga através de técnicas de programação linear, feita anteriormente, pode gerar ciclos nos caminhos. A re-otimização da solução usando o número total de hops (21) da rede como uma nova função objetivo pode eliminar essas "anamolias". Além disso, a coloração (alocação de comprimento de onda) também pode ser feita nesse passo. Logo, nós adicionamos as equações (14)-(19). Nós assumimos uma certa disponibilidade de número de comprimentos de onda $W$ e obtemos o número mínimo necessário desses $W$, o Wmin, para colorir os caminhos ópticos.

\subsection{RESULTADOS NUMÉRICOS}

Considere a matriz de tráfego $\mathrm{T}=\lambda^{s d}$ da Tabela 1, obtida pela geração de números aleatórios entre 0 e 1 com uma distribuição gaussiana, com $\mu=0.5$ e $\sigma=0.1$. Temos $W=2$.

Nós iremos obter as matrizes das tabelas 2 e 3, de links virtuais $b_{i j}$, para graus virtuais 1 e 2 .

\begin{tabular}{c|cccccc}
\hline \hline$\lambda^{\text {sd }}$ & 0 & 1 & 2 & 3 & 4 & 5 \\
\hline 0 & - & 0,90 & 0,62 & 0,51 & 0,28 & 0,52 \\
1 & 0,53 & - & 0,39 & 0,92 & 0,26 & 0,15 \\
2 & 0,47 & 0,31 & - & 0,34 & 0,21 & 0,14 \\
3 & 0,29 & 0,48 & 0,34 & - & 0,99 & 0,36 \\
4 & 0,15 & 0,44 & 0,14 & 0,84 & - & 0,99 \\
5 & 0,48 & 0,19 & 0,99 & 0,75 & 0,18 & - \\
\hline \hline
\end{tabular}

Tabela 1. Matriz de Tráfego.

\begin{tabular}{c|cccccc}
\hline \hline$b_{i j}$ & 0 & 1 & 2 & 3 & 4 & 5 \\
\hline 0 & - & 1 & - & - & - & - \\
1 & - & - & - & 1 & - & - \\
2 & 1 & - & - & - & - & - \\
3 & - & - & - & - & 1 & - \\
4 & - & - & - & - & - & 1 \\
5 & - & - & 1 & - & - & - \\
\hline \hline
\end{tabular}

Tabela 2. Grau virtual 1.

A Figura 4 mostra os ciclos formados pelo grau virtual " 1 ". Isto justifica a necessidade de re-otimização proposta previamente no passo 3. Além disso, os caminhos mais curtos são escolhidos, por exemplo $b_{34}$ necessita de somente um hop 


\begin{tabular}{c|cccccc}
\hline \hline$b_{i j}$ & 0 & 1 & 2 & 3 & 4 & 5 \\
\hline 0 & - & 2 & - & - & - & - \\
1 & - & - & - & 2 & - & - \\
2 & 2 & - & - & - & - & - \\
3 & - & - & - & - & 2 & - \\
4 & - & - & - & - & - & 2 \\
5 & - & - & 2 & - & - & - \\
\hline \hline
\end{tabular}

Tabela 3. Grau virtual 2.

físico depois da re-otimização. Esses dois fatores garantem um mínimo número de hops físicos na rede. Logo, o sucesso da re-otimização é garantido.

Na Figura 5 os comprimentos de onda são mostrados separados, como sub-redes, para melhor visualização. Por exemplo, há dois caminhos ópticos paralelos do nó 0 para o nó 1 , implicando em $b_{011}=1 \mathrm{e} b_{012}=1$.

Na Figura 6 nós observamos o número mínimo de comprimentos de onda obtidos em função do grau virtual e comparamos com o lower bound deste número, valor obtido teoricamente de [6] o qual é dado por $W \geq\lceil\Delta / \delta p\rceil$. Onde $\delta p$ é o grau físico da topologia física (número de fibras que estão conectadas ao nó). Se o número de comprimentos de onda disponíveis for menor que o número mínimo requerido, Wmin, então a solução não é viável.
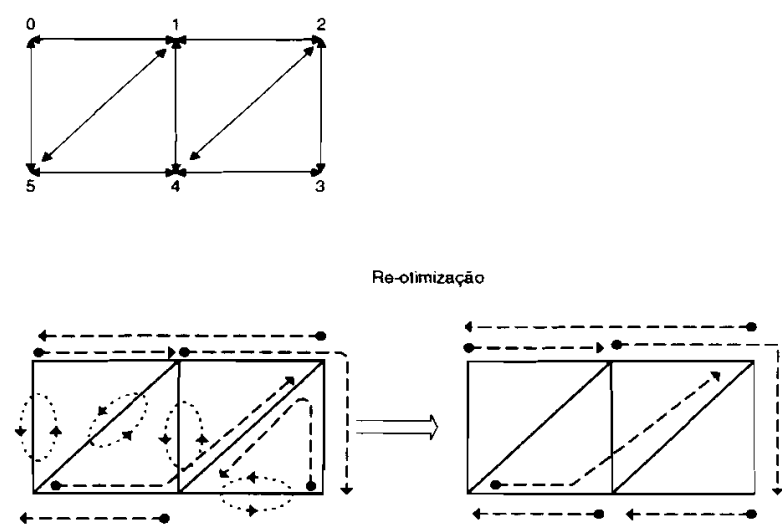

Figura 4. Otimização para grau virtual 1.

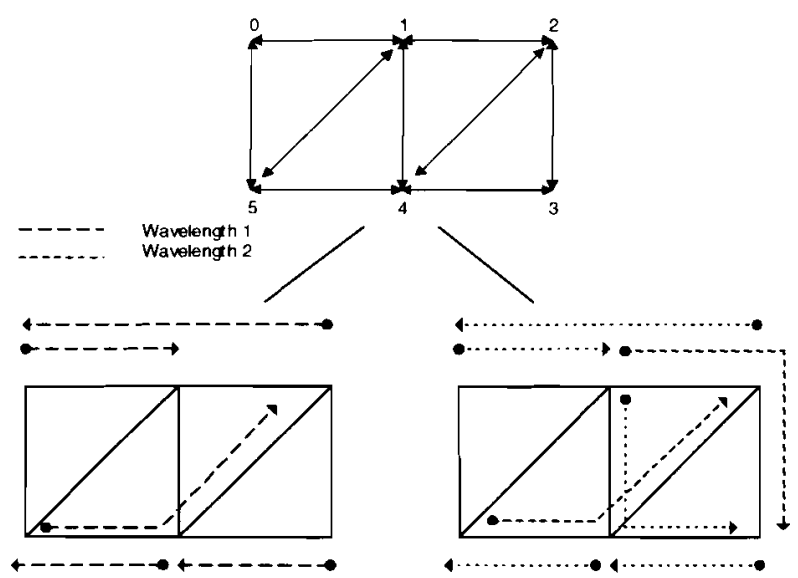

Figura 5. Otimização para grau virtual 2.
Na Figura 7 nós observamos a eficiência da re-otimização para eliminar os ciclos da rede, com diversos graus virtuais, e encontrar caminhos mais curtos para os caminhos ópticos, diminuindo significamente o número de hops físicos na rede.

Todos os resultados, incluindo a máxima carga numa fibra, são sumarizados na Tabela 4.

\begin{tabular}{|c||c|c|c|c|}
\hline$\Delta$ & $L$ & $W_{\min }$ & $H L$ & $H L / O$ \\
\hline \hline $\mathbf{1}$ & 1 & 1 & 9 & 18 \\
\hline $\mathbf{2}$ & 2 & 2 & 18 & 27 \\
\hline $\mathbf{3}$ & 2 & 2 & 32 & 34 \\
\hline $\mathbf{4}$ & $\mathbf{3}$ & 3 & 41 & 51 \\
\hline $\mathbf{5}$ & 4 & 5 & 50 & 72 \\
\hline
\end{tabular}

Tabela 4. Resultados para a rede de 6 nós.

Onde:

- $\Delta$ : Grau virtual

- L: Carga máxima em um link de fibra

- Wmin: Número mínimo de comprimentos de ondas necessários

- HL: Número total de hops físicos com reotimização

- $H L / O$ : número total de hops físicos sem reotimização.

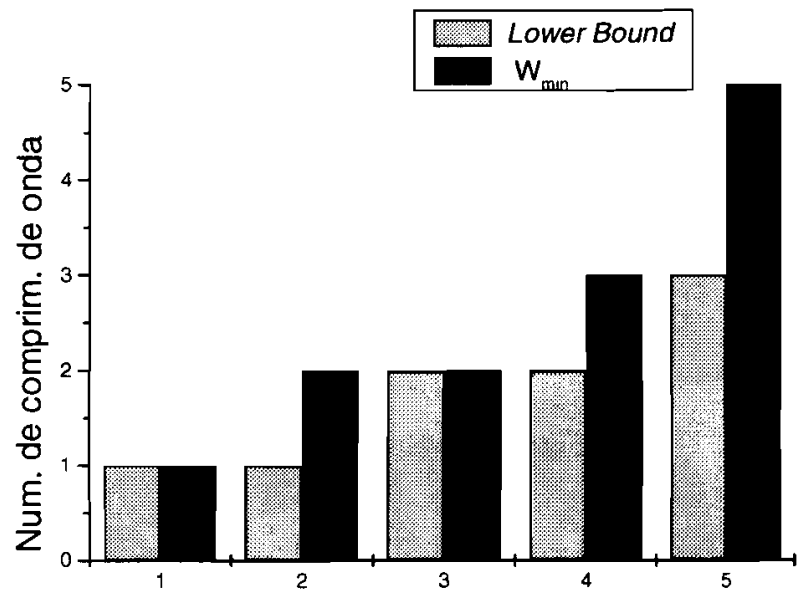

Grau virtual

Figura 6. Comparação entre o lower bound e o número mínimo de wavelengths, para diversos graus virtuais, encontrados com a heurística MinW.

\subsection{RESULTADOS NUMÉRICOS PARA UMA REDE DE GRANDE DIMENSÃO (REDE HIPOTÉTICA BRASILEIRA)}

Nesta seção, nós mostramos os resultados obtidos para uma rede de grande dimensão. A chamada rede hipotética brasileira (Figura 8), que tem 12 nós e 20 pares de links físicos unidirecionais. A matriz de tráfego foi gerada aleatoriamente de uma distribuição uniforme entre 0 e l (Tabela 5). 


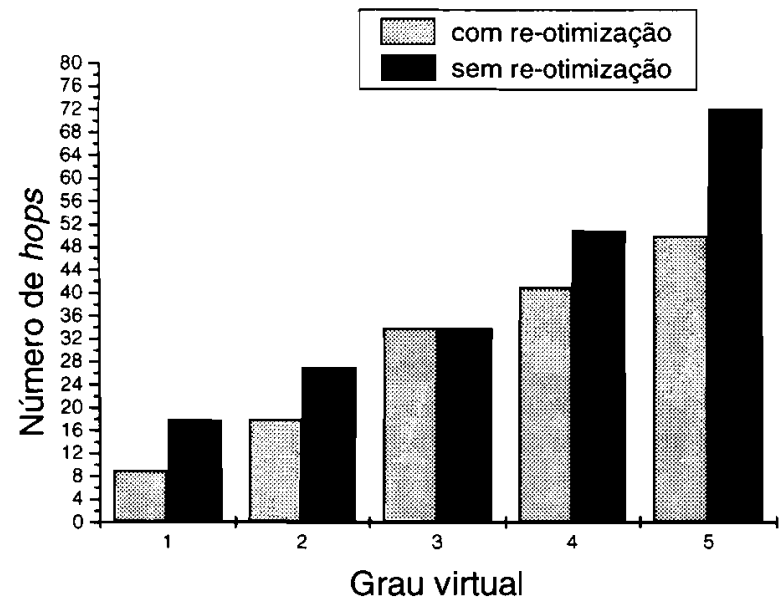

Figura 7. Número de hops, para diversos graus virtuais, com e sem reotimização.

A simulação da estratégia proposta para redes de grande dimensão é importante, pois mostra sua eficiência em "bloquear" a complexidade do problema. A Tabela 6 mostra os resultados com a re-otimização. Note que a complexidade do VTD é bloqueada devida a heurística HVTD, a complexidade do passo 3 do PTD é bloqueada devido a re-otimização com mínimo número de hops (o programa enxergará apenas caminhos mais curtos).

Usando o software de otimização CPLEX(C) [9] num Intel Pentium IV/1.6Ghz, o tempo de simulação ainda é um gargalo, saindo da escala de minutos, no passo 2 , pois a minimização da carga acarreta tempos de simulação na ordem de horas.

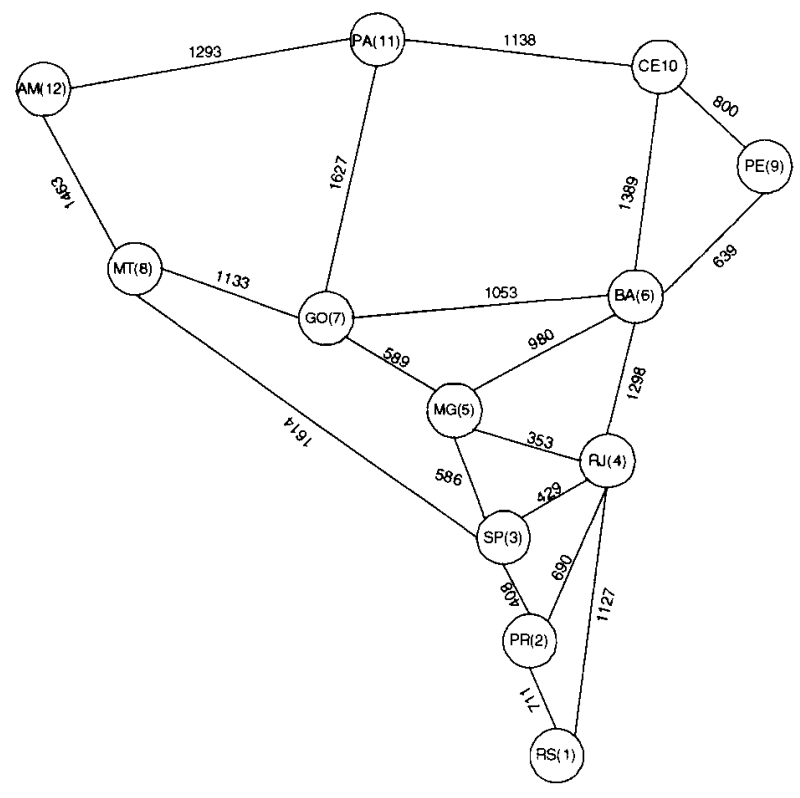

Figura 8. Rede hipotética brasileira.

para a rede de grande dimensão em estudo. Para a nossa rede de grande dimensão a simulação foi programada para parar necessariamente em $\mathrm{l}$ h (o que pode provocar uma degradação no valor da função objetivo). Entretanto, mesmo essa com-

$\begin{array}{llllllllllll} & \mathbf{0 , 9 2} & \mathbf{0 , 3 2} & \mathbf{0 , 8 4} & \mathbf{0 , 3 0} & \mathbf{0 , 4 9} & \mathbf{0 , 8 3} & \mathbf{0 , 1 7} & \mathbf{0 . 2 8} & \mathbf{0 , 5 2} & \mathbf{0 , 4 1} & \mathbf{0 , 3 2} \\ \mathbf{0 , 2 3} & & \mathbf{0 , 2 0} & \mathbf{0 , 5 2} & \mathbf{0 , 2 9} & \mathbf{0 , 8 9} & \mathbf{0 , 5 6} & \mathbf{0 , 9 7} & \mathbf{0 , 4 6} & \mathbf{0 , 6 4} & \mathbf{0 , 3 0} & \mathbf{0 , 9 6} \\ \mathbf{0 , 6 0} & \mathbf{0 . 1 7} & & \mathbf{0 , 2 0} & \mathbf{0 , 1 9} & \mathbf{0 , 8 2} & \mathbf{0 , 3 7} & \mathbf{0 , 2 7} & \mathbf{0 , 0 6} & \mathbf{0 , 2 0} & \mathbf{0 , 8 7} & \mathbf{0 , 7 2} \\ \mathbf{0 , 4 8} & \mathbf{0 , 4 0} & \mathbf{0 , 4 0} & & \mathbf{0 , 6 8} & \mathbf{0 , 6 4} & \mathbf{0 , 7 0} & \mathbf{0 , 2 5} & \mathbf{0 , 9 8} & \mathbf{0 , 3 7} & \mathbf{0 , 0 1} & \mathbf{0 , 4 1} \\ \mathbf{0 , 8 9} & \mathbf{0 , 9 3} & \mathbf{0 , 2 7} & \mathbf{0 , 8 3} & & \mathbf{0 , 8 1} & \mathbf{0 , 5 4} & \mathbf{0 , 8 7} & \mathbf{0 , 5 8} & \mathbf{0 , 7 8} & \mathbf{0 , 7 6} & \mathbf{0 , 7 4} \\ \mathbf{0 , 7 6} & \mathbf{0 , 9 1} & \mathbf{0 , 1 9} & \mathbf{0 , 0 1} & \mathbf{0 , 5 4} & & \mathbf{0 , 4 4} & \mathbf{0 , 7 3} & \mathbf{0 , 4 2} & \mathbf{0 , 6 8} & \mathbf{0 , 9 7} & \mathbf{0 , 2 6} \\ \mathbf{0 , 4 5} & \mathbf{0 , 4 1} & \mathbf{0 , 0 1} & \mathbf{0 , 6 8} & \mathbf{0 , 1 5} & \mathbf{0 , 3 4} & & \mathbf{0 , 1 3} & \mathbf{0 , 5 1} & \mathbf{0 , 4 6} & \mathbf{0 , 9 9} & \mathbf{0 , 4 3} \\ \mathbf{0 , 0 1} & \mathbf{0 , 8 9} & \mathbf{0 , 7 4} & \mathbf{0 , 3 7} & \mathbf{0 , 6 9} & \mathbf{0 , 2 8} & \mathbf{0 , 6 2} & & \mathbf{0 . 3 3} & \mathbf{0 , 5 6} & \mathbf{0 , 7 8} & \mathbf{0 , 9 3} \\ \mathbf{0 , 8 2} & \mathbf{0 , 0 5} & \mathbf{0 , 4 4} & \mathbf{0 , 8 3} & \mathbf{0 , 3 7} & \mathbf{0 , 3 4} & \mathbf{0 , 7 9} & \mathbf{0 , 8 9} & & \mathbf{0 , 7 9} & \mathbf{0 , 4 3} & \mathbf{0 , 6 8} \\ \mathbf{0 , 4 4} & \mathbf{0 , 3 5} & \mathbf{0 , 9 3} & \mathbf{0 , 5 0} & \mathbf{0 , 8 6} & \mathbf{0 , 5 3} & \mathbf{0 , 9 5} & \mathbf{0 , 1 9} & \mathbf{0 , 2 2} & & \mathbf{0 , 4 9} & \mathbf{0 , 2 1} \\ \mathbf{0 , 6 1} & \mathbf{0 , 8 1} & \mathbf{0 , 4 6} & \mathbf{0 , 7 0} & \mathbf{0 , 8 5} & \mathbf{0 , 7 2} & \mathbf{0 , 5 2} & \mathbf{0 , 2 9} & \mathbf{0 , 5 7} & \mathbf{0 , 6 0} & & \mathbf{0 , 8 3} \\ \mathbf{0 , 7 9} & \mathbf{0 , 0 1} & \mathbf{0 , 4 1} & \mathbf{0 , 4 2} & \mathbf{0 . 5 9} & \mathbf{0 , 3 0} & \mathbf{0 , 8 8} & \mathbf{0 , 6 6} & \mathbf{0 , 7 6} & \mathbf{0 , 0 5} & \mathbf{0 , 6 4} & \\ & & & & & & & & & & & \end{array}$

Tabela 5. Matriz de tráfego para a rede hipotética brasileira.

plexidade do passo 2 pode ser aliviada se resolvermos os passos 2 e 3 juntamente (função multiobjetivo), desde que sejam atribuídos pesos adequados a cada função objetivo (no caso em estudo carga e número de hops). Neste caso a complexidade seria aliviada porque a minimização da carga ocorreria com um roteamento por caminhos mais curtos e não adaptativo.

\begin{tabular}{|c||c|c|c|}
\hline$\Delta_{l}$ & $L$ & $W_{\min }$ & $H L$ \\
\hline \hline $\mathbf{1}$ & 1 & 1 & 32 \\
\hline $\mathbf{2}$ & 2 & 2 & 52 \\
\hline $\mathbf{3}$ & 3 & 3 & 78 \\
\hline $\mathbf{4}$ & 4 & 4 & 104 \\
\hline $\mathbf{5}$ & 4 & 4 & 130 \\
\hline $\mathbf{6}$ & 5 & 5 & 147 \\
\hline
\end{tabular}

Tabela 6. Resultados para a rede de 12 nós.

\section{COMENTÁRIOS E CONCLUSÕES}

Neste artigo nós propomos uma estratégia iterativa através de Programação Linear Inteira para resolver os problemas de projeto da topologia virtual e topologia física das redes ópticas de forma integrada. Algumas formulações podem ser trocadas por heurísticas convenientes de maneira a diminuir a complexidade computacional.

A solução do problema VTD gera uma requisição para um conjunto de caminhos que devem ser roteados na topologia física. Estes caminhos ópticos serão então roteados de maneira a minimizar a máxima carga permitida em uma fibra. No entanto, isto pode gerar ciclos na rede. Esses ciclos podem ser eliminadas por re-otimização da solução com uma nova função objetivo no PTD: o mínimo número de hops, com o mínimo valor da carga máxima obtida no critério de otimização anterior. A fase final é a alocação de comprimentos de onda para os caminhos ópticos.

A estratégia foi apresentada e testada para dois cenários de rede, confirmando a eficiência da proposta para os critérios de otimização adotados (carga, mínimo número de hops e mínimo número de comprimentos de onda) e tempo computacional.

\section{AGRADECIMENTOS}

Os autores gostariam de agradecer a FAPESP, CNPq e a Ericsson Telecomunicações do Brasil S.A. 


\section{REFERÊNCIAS}

[1] M.S. Alencar, Telefonia Digital, Editora Érica, São Paulo, 1998.

[2] H. Waldman, M.D. Yacoub, Telecomunicações: Princípios e Tendências, Editora Érica, São Paulo, 1997.

[3] K.G. Coffman and A.M. Odlyzko, "Internet growth: Is there a "Moore Law" for data traffic?" Handbook of Massive Data Sets, J. Abello, P.M Pardalos, and M. G. C. Resende, eds., Kluwer, 2001.

[4] R. Ramaswami and K.N. Sivarajan, Optical Networks: a Practical Perspective, Morgan Kauffmann Publishers, 1998.

[5] C. Siva Ram Murthy and Mohan Gurusamy, WDM Optical Networks: Concepts, Design, and Algorithms, Prentice Hall, 2002. ISBN 0-13-060637-5.

[6] R. Ramaswami and K.N.Sivarajan, "Design of logical topologies for wavelength-Routed All Optical Networks", IEEE/JSAV, vol. 14, pp. 840-851, june 1996.

[7] R. Ramaswami and K. N. Sivarajan, "Routing and wavelength assignment in all-optical networks", IEEE/ACM Trans. Networking, vol. 3, n.5, pp. 489-500, Oct. 1995.

[8] D. Banerjee and B. Mukherjee, 1996 "Pratical approaches for routing and wavelength Wavelength assignment in large all-optical wavelength-routed networks." IEEE Journal on Selected Areas in Communications, 14 (5): 903-908, june 1996.

[9] CPLEX Optimization Software. [Online]. Available: http://www.ilog.com.

[10] R. M. Krishnaswamy and K.N. Sivarajan "Design of Logical Topologies: A Linear Formulation for Wavelength-Routed Optical Networks with No Wavelength Changers" IEEE/ACM Transactions on Networking, vol. 9, NO.2, April 2001

[11] R. Dutta, G.N.Roukas, "Survey of Virtual Topology Design Algorithms for Wavelength-routed Optical Networks", Optical Networks, Vol.1, pp.73-88, janeiro 2000

[12] H. Zang, J.P. Jue and B. Mukherjee, "A Review of Routing and Wavelength Assignment Approaches for Wavelength-Routed optical WDM Networks", Optical Networks Magazine, vol.1, pp. 47-60, Jan. 2000

[13] W.Y. John. "Advanced in the management and control of optical Internets", IEEE J. Select. Areas Communic., vol 20, pp. 768-785, may 2002.

[14] K. D. R. Assis, H. Waldman. "An Integrated Design for Topolologies of Optical Networks, part II: Resources of Wavelength Conversion" SBrT Simpósio Brasileiro de Telecomunicações, Outubro, 5-8, 2003. Rio de Janeiro, RJ, Brasil.

[15] K. D. R. Assis, H. Waldman "An Integrated Design for Topolologies of Optical NetworksIEEE/SBrT International Telecommunication Symposium, September 8-12, 2002. Natal, RN, BrazilProceedings of IEEE/ITS' 02 .
Karcius D. R. Assis graduou-se em engenharia elétrica em 1997 pela Universidade Federal da Paraíba (UFPB/Campina Grande). Recebeu o título de mestre em Eng. Elétrica pela Universidade Federal do Espírito Santo (UFES) em 2000 e o de Doutor em Eng. Elétrica pela Universidade Estadual de Campinas (UNICAMP) em 2004 Durante o doutorado ele foi pesquisador do Optical Networking Lab, (Optinet) da FEEC/UNICAMP. Em agosto de 2004 Karcius juntouse à Universidade Salvador (UNIFACS), onde é professor do curso de Ciência da Computação e pesquisador do NUPERC (Núcleo Interdepartamental de Pesquisas em Redes de Computadores) onde tem dado continuidade ao desenvolvido de pesquisas sobre planejamento e otimização de redes ópticas.

Helio Waldman nasceu em São Paulo, SP, em 20 de junho de 1944. Graduou-se Engenheiro de Eletrônica pelo Instituto Tecnológico de Aeronáutica (ITA) de São José dos Campos, SP em 1966, e recebeu os títulos de M. Sc. e Ph. D. da Universidade de Stanford na California, USA, em 1968 e 1972 respectivamente, ambos em Engenharia Elétrica. Após dois anos na COPPE/UFRJ como Professor Adjunto, juntou-se à Universidade Estadual de Campinas (UNICAMP), onde é Professor Titular desde 1980. Em 1980, foi nomeado Diretor Associado da Faculdade de Engenharia de Campinas (FEC/UNICAMP). Em 1982, foi nomeado Diretor da mesma Faculdade, cargo que ocupou até 1986. De 1986 a 1990, foi Pró-Reitor de Pesquisa da UNICAMP. O Dr. Waldman é "Senior Member" do IEEE desde 1990, e Membro Sênior da Sociedade Brasileira de Telecomunicações (SBrT), onde serviu como Presidente entre 1988 e 1990. Em 1996, foi agraciado pela Unicamp com a Bolsa de Reconhecimento Acadêmico "Zeferino Vaz", "por ter se destacado nas suas atividades de Docência e Pesquisa durante o ano de 1995, entre os docentes da Faculdade de Engenharia Elétrica". Foi também: Editor da Revista da Sociedade Brasileira de Telecomunicações de 1992 a 1996; e Presidente da Comissão Permanente de Dedicação Integral à Docência e Pesquisa (CPDI) da UNICAMP de outubro de 2001 a abril de 2002. Desde 1973, o Prof. Waldman tem pesquisado ativamente os sistemas de Comunicações Digitais. A partir dos anos 80 seus interesses de pesquisa têm focalizado o canal de fibra óptica. É autor ou co-autor de três livros: "Processamento de Sinais Digitais" (1987), "Fibras Ópticas: Tecnologia e Projeto de Sistemas" (1991), e "Telecomunicações: Princípios e Tendências" (1997). Publicou quinze artigos em periódicos científicos especializados estrangeiros com corpo editorial. Apresentou 56 comunicações em congressos e simpósios ( 30 no Brasil e 26 no Exterior), das quais 51 foram publicadas em anais na forma de trabalhos completos. Orientou 31 Teses, sendo 26 de Mestrado e cinco de Doutorado. Seus interesses atuais de pesquisa estão nas áreas de Redes Ópticas, Enlaces Ópticos Digitais, e Redes IP sobre WDM, áreas nas quais tem orientado Teses e ministrado disciplinas de pós-graduação. No curso de graduação em Engenharia Elétrica, tem ministrado disciplinas de Eletromagnetismo, Ondas Guiadas e Comunicações Ópticas. Está interessado também em desenvolver a reflexão sobre as novas tecnologias de Telecomunicações e seus impactos sobre as relações de trabalho, o processo educacional e a sociedade. 